\title{
ANÁLISE VISUAL DA EVOLUÇÃO DE COLEÇÕES DE DOCUMENTOS UTILIZANDO TAG CLOUD
}

\section{VISUAL ANALYSIS OF DOCUMENT COLLECTIONS EVOLUTION USING TAG CLOUD}

André Luis Dias Andreotti ${ }^{1}$; Danilo de Medeiros Eler ${ }^{1}$; Ives Renê Venturini Pola ${ }^{1}$; Almir Olivette Artero ${ }^{1}$;

Faculdade de Ciências e Tecnologia - Universidade Estadual Paulista "Júlia de Mesquita Filho" (FCT/UNESP) ${ }^{1}$

dias.andreotti@gmail.com;_daniloeler@fct.unesp.br; ivesrene@gmail.com;

almir@fct.unesp.br;

RESUMO - Nos dias atuais, há uma grande quantidade de informações disponíveis em diversas coleções de documentos, dentre eles artigos, teses, e outros trabalhos acadêmicos. Coleções de artigos científicos podem representar a trajetória acadêmica de um pesquisador em particular, e a evolução de uma linha de pesquisa com o passar dos anos. Neste trabalho, são investigadas técnicas de exploração e de visualização de documentos, com foco na técnica tag cloud. Para tanto, foi desenvolvida uma ferramenta que auxilia na visualização de coleções de documentos, possibilitando verificar a evolução da coleção ao longo do tempo, quanto o surgimento de novos tópicos ou quanto ao seu conteúdo, comparando a nuvem de etiquetas que foi gerada para cada documento.

Palavras-chave: tag cloud; documentos; evolução.

ABSTRACT - Nowadays, there is a lot of information available in various document collections, including articles, theses, and another scholarly works. Collections of papers may represent the academic career of a researcher in particular, and the development of a research line over the years. In this work, we investigated techniques of exploration and visualizations of documents, focusing on tag cloud technique. Therefore, a tool was developed to aid in document collection visualization and exploration, enabling to check the collection evolution over time, regarding to the emergence of new topics or their content, comparing distinct tag clouds generated for each document.

Keywords: tag cloud; documents; evolution. 


\section{INTRODUÇÃO}

Atualmente, existe um amplo volume de dados armazenados por diversos meios eletrônicos. A exploração e análise desse vasto volume de dados está se tornando cada vez mais difícil, devido à grande quantidade de dados e de atributos. Por isso, muita pesquisa tem sido realizada em técnicas que apoiem os usuários no entendimento desses dados. Uma das áreas de pesquisa que tem se destacado nos últimos anos é a análise visual de dados. Na literatura, há um grande número de técnicas de visualização de informação que foram desenvolvidas ao longo da última década para apoiar a exploração de grandes conjuntos de dados. Entretanto, a habilidade do ser humano de armazenar dados aumenta a uma velocidade mais rápida do que a capacidade de analisálos e, se nenhuma informação for extraída, o armazenamento terá sido inútil, afirma Keim (2002).

Um exemplo de conjunto de dados que tem crescido nos últimos anos são as coleções de artigos científicos, as quais podem representar a trajetória acadêmica de um pesquisador em particular, e a evolução de uma linha de pesquisa com o passar dos anos. Assim, analisar a evolução temporal dos assuntos abordados em coleções como estas é de extrema importância para acadêmicos e pesquisadores em geral, pois, podem avaliar as tendências da área que pretendem estudar e tomarem decisões a partir desta avaliação. Desta forma, é notável o quão útil é propor ferramentas que auxiliem nesta análise, facilitando a exploração textual.

Este trabalho trata essencialmente do estudo e aplicação da técnica de Visualização de Informação tag cloud, visando a exploração textual de uma coleção de documentos na busca pela evolução temporal de seus tópicos, tais como: identificação de emergências de tópicos em instantes específicos de tempo; a especialização de um tópico; e o desaparecimento de tópicos. Essa evolução temporal pode ser detectada por meio da identificação de termos mais relevantes de cada documento, analisando as nuvens de etiquetas geradas.

Neste trabalho foi desenvolvida a ferramenta TGEE (Tag Clouds Enviroment Exploration), com objetivo de facilitar a análise desse amplo volume de dados textuais.

O restante deste trabalho está organizado em cinco seções, na Seção 2 é realizada uma breve contextualização da área estudada para o desenvolvimento do presente trabalho. Na Seção 3 é descrita a abordagem adotada para o desenvolvimento da ferramenta TGEE (Tag Clouds Enviroment Exploration). Na Seção 4 são descritos os 
experimentos realizados e os resultados obtidos são exibidos. Finalmente, na Seção 5 as considerações finais e conclusões são apresentadas, bem como sugestões de aperfeiçoamento e trabalhos futuros.

\section{FUNDAMENTAÇÃO TEÓRICA}

A Visualização de Informação é uma área da Ciência da Computação que tem por objetivo dar sentido aos dados por meio de representações gráfica, com o intuito de ampliar a capacidade cognitiva do ser humano em processos de exploração de dados, conforme afirmam Card, Mackinlay e Shneidermann (1999), Chen (2006) e Spence (2007). Uma das técnicas da Visualização de Informação é a tag cloud (nuvem de etiquetas), a qual consiste na criação de uma nuvem de palavras, que pertençam a um documento, fazendo a distinção entre elas pelo tamanho da fonte ou cor. Assim, na representação gráfica gerada, as palavras são destacadas levando em consideração a quantidade de vezes que aparecem no texto, por intermédio da tokenização e contabilização de cada termo. Na Figura 1 são apresentadas duas visualizações geradas por meio da técnica tag cloud. As duas visualizações foram geradas pelo site tag crowd, cuja URL é www.tagcrowd.com. A visualização da esquerda foi processada utilizando o texto do discurso realizado pelo presidente dos Estados Unidos da América,
George W. Bush, no ano de 2002. E a outra visualização sobre o discurso realizado pelo, até então, atual presidente Barack Obama em 2011.

Palavras que foram mais pronunciadas pelos respectivos presidentes, foram destacadas nesta representação pelo tamanho da fonte e intensidade da cor azul. A partir da imagem é possível observar os assuntos que eram prioridade na época, em 2002, logo após o atentado terrorista de 11 de setembro de 2001, onde destacam as palavras "american", "terrorists", "security" e "afghanistan". Por meio dessa representação gráfica, é possível observar a troca de contexto nos assuntos políticos, quase dez anos após o discurso anterior.

Figura 1. Tag cloud comparando o discurso realizado pelos dois presidentes dos Estados Unidos da América, no ano de seus respectivos mandatos.

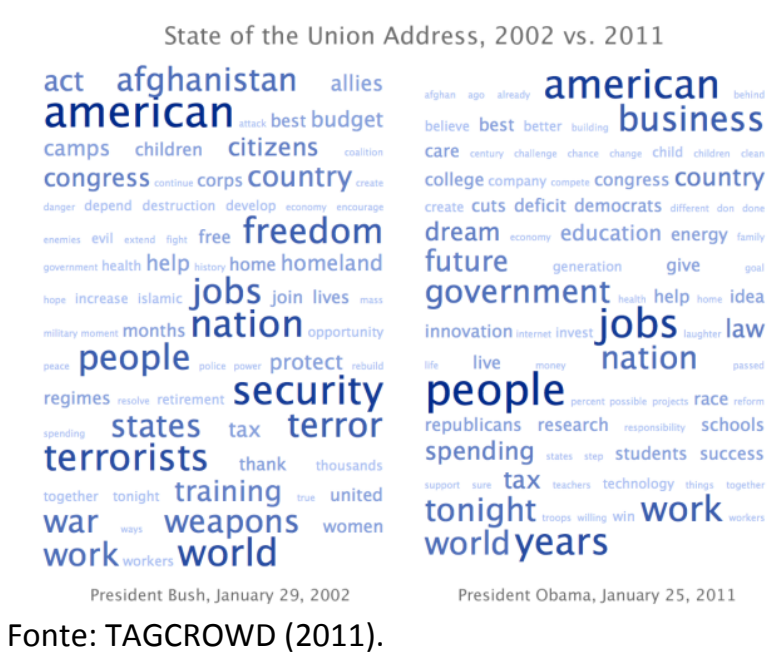


A partir da visualização é possível ter uma ideia geral do assunto tratado nos dois discursos e as principais diferenças entre eles, sem que seja necessário ler todo o discurso. Apesar de parecer simples a ideia de que documentos distintos possuam distribuições de termos diferentes, esta hipótese mostrou-se bem-sucedida na classificação de textos em assuntos, mostrando desempenho superior ao de representações mais complexas, reforça Sebastiani (2002).

A Figura 2 mostra outro exemplo de visualização que utiliza tag cloud. Nesta representação a mudança positiva do preço da ação é indicada pela cor verde, a mudança negativa pela cor vermelha, e o tamanho da fonte indica a variação percentual.

Figura 2. Movimento dos preços das ações.

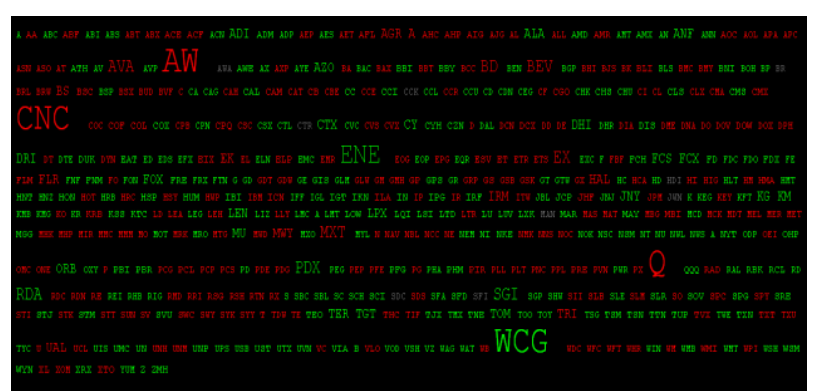

Fonte: Warrena (2007).

Um exemplo que utiliza tag cloud para representar dados quantitativos está expresso na Figura 3, onde a população mundial foi distinguida pelo tamanho da fonte do nome do país.
Figura 3. População de cada um dos países do mundo. Criada em $\mathrm{R}$ (linguagem de programação) utilizando wordcloud.

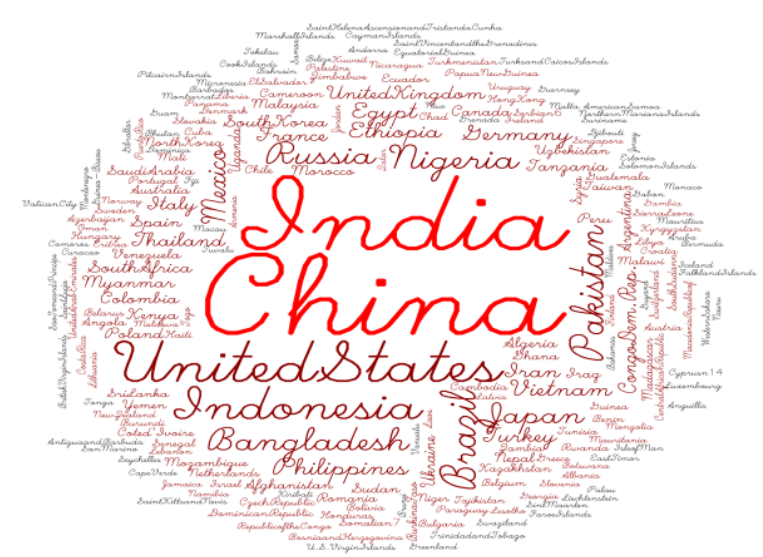

Fonte: http://www.wordclouds.com

\section{ABORDAGEM PROPOSTA}

O processo de desenvolvimento da ferramenta TGEE (Tag Clouds Enviroment Exploration), fruto do presente trabalho, teve sua base nas etapas de pré-processamento e representação de coleções de documentos utilizadas por Alencar (2013), sendo elas: tokenização, remoção de stopwords, Stemming, Lei de Zipf e Corte de Luhn. A ferramenta foi desenvolvida utilizando a linguagem de programação Java, visando a garantia de portabilidade para sistemas operacionais que suportam a máquina virtual Java (Java Virtual Machine - JVM).

A Figura 4 descreve a abordagem proposta, mostrando cada etapa do processamento realizado, partindo da aquisição da coleção de documentos, passando pela tokenização dos termos, remoção de palavras comuns e irrelevantes 
para a coleção analisada, contagem dos termos no modelo vetorial, utilização das bibliotecas WordCram e OpenCloud para, finalmente, gerar a visualização da tag cloud.

Figura 4. Etapas do processo da abordagem proposta.

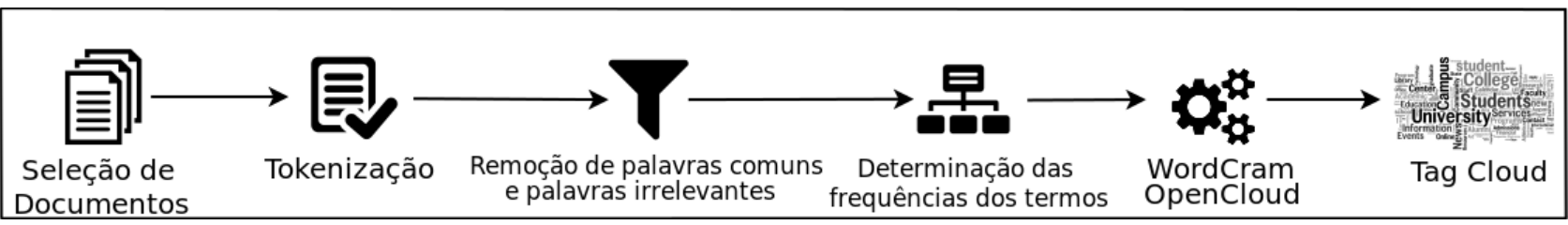

Fonte: (Própria).

Foram utilizadas duas bibliotecas responsáveis por gerar a visualização das tag clouds, sendo elas: WordCram e OpenCloud. WordCram é um trabalho, disponível na URL wordcram.org, que utiliza a linguagem de programação Processing de código aberto e ambiente de desenvolvimento integrado, construído para as artes eletrônicas e comunidades de projetos visuais. OpenCloud auxilia na projeção dos termos horizontalmente no plano e em ordem alfabética, sendo menos sofisticada que a primeira, disponível na URL www.sourceforge.net/projects/opencloud/.

\subsection{SELEÇÃO DE DOCUMENTOS}

Visando a praticidade, no processamento de coleções de documentos pelo usuário final da ferramenta desenvolvida, foi implementada a possibilidade de seleção de vários arquivos textos, no formato " $t x t$ " (formato de arquivo somente texto, sem formatação), sendo possível optar por gerar uma tag cloud para cada arquivo selecionado, ou concatenar todo o texto dos arquivos e gerar apenas uma tag cloud.

\subsection{TOKENIZAÇÃO}

A primeira etapa a ser realizada é a tokenização do texto, separando-o em tokens. Caso o texto esteja devidamente estruturado, de acordo com a pontuação da linguagem utilizada, esta tarefa torna-se fácil, selecionando as palavras quando espaços ou caracteres de pontuação são encontrados. A Figura 5 exibe um exemplo deste processo.

Figura 5. Exemplo de tokenização de uma frase.

\footnotetext{
Entrada: "Você não pode juntar os pontos olhando para frente; você pode conectá-los apenas olhando para trás".

Saída: Você não pode juntar os pontos olhando para frente você pode conectá-los apenas olhando para trás

Fonte: (Própria).
}

\subsection{STOPWORDS}

Uma etapa fundamental é a de verificar quais palavras representam melhor o assunto de um texto, normalmente, substantivos. 
Assim, é necessário remover os termos irrelevantes, tais como artigos, adjetivos, advérbios, conjunções e preposições. Esses termos são conhecidos como stopwords e são dependentes da linguagem adotada no documento. Esse processo é feito por meio de listas pré-estabelecidas de stopwords da linguagem do documento processado.

\subsection{Lei de Zipf e Cortes de Luhn}

Deve-se considerar que termos que aparecem com muita frequência em vários documentos e outros que aparecem em poucos documentos podem ser desconsiderados no processamento, pois, não ajudam a diferenciar a semântica de uma coleção de documentos.

A Lei de Zipf (1949) leva em consideração o número de incidências de termos, e traça uma razão em comum entre eles. Considerando o histograma de frequência dos termos de uma coleção de documentos ordenado em ordem crescente obtém-se a "Curva de Zipf" observada na Figura 6. O eixo cartesiano " $r$ " representa os termos, em ordem decrescente de frequência, e o eixo cartesiano " $f$ " representa a frequência desses termos.
Figura 6. Curva de Zipf.

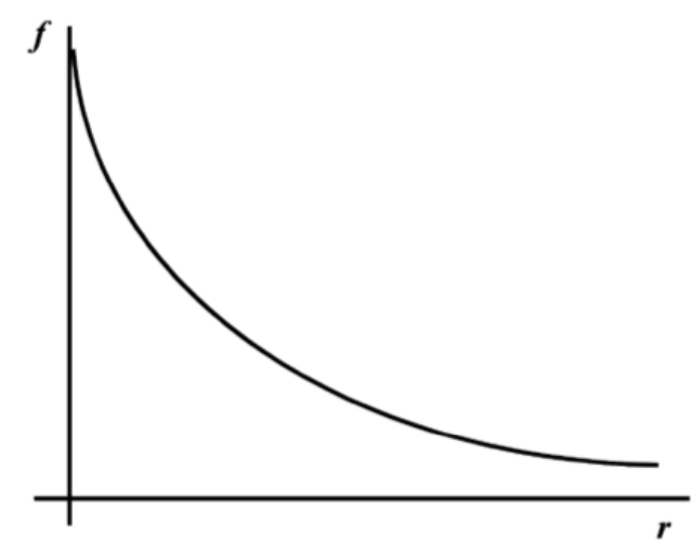

Fonte: Zipf (1949).

Luhn (1958), usou a Lei de Zipf para especificar dois pontos de corte, os quais denominou de superior e inferior, para separar os termos relevantes, observados na Figura 7. Desta forma, os termos que aparecem com muita frequência em vários documentos e outros que aparecem em poucos documentos, são desconsiderados. Com base neste conhecimento, a ferramenta TGEE considera apenas os termos mais significativos quando um documento é processado, diminuindo o esforço computacional necessário. É possível adicionar termos a uma lista de remoção, ignorando os que possuem uma alta frequência já esperada na coleção que será avaliada, por exemplo, ao processar uma coleção de documentos da área de Visualização de Informação, os termos: “informação" e "visualização" tendem a aparecer com uma alta frequência em todos os textos desta coleção, logo esses termos podem ser adicionados à lista de remoção, 
pois, os mesmos não servirão para analisar a evolução temporal entre os documentos.

Figura 7. Cortes de Luhn.

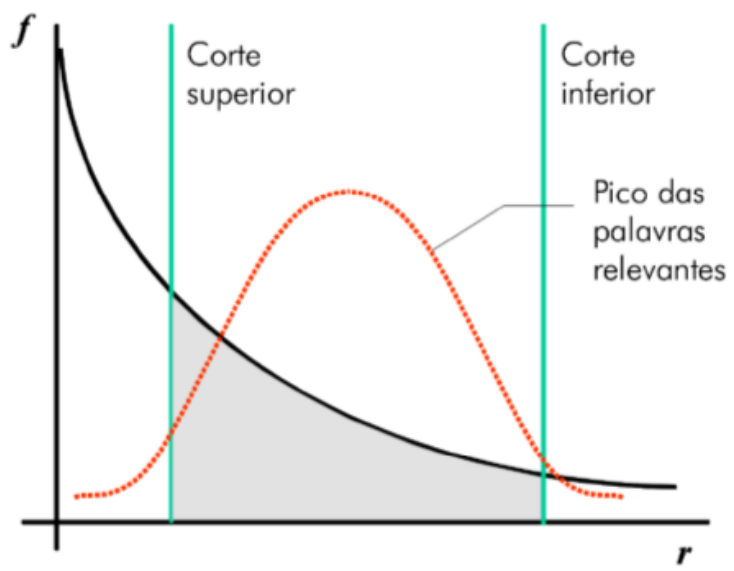

Fonte: Luhn (1958).

\subsection{TAG CLOUDS}

O modelo vetorial, descrito em Salton, Wong e Yang (1975), também conhecido como bag-of-words, descreve cada documento como um vetor de frequências dos termos que nele ocorrem. Sendo $D=\left\{d_{1}\right.$ $\left.d_{2}, \ldots, d_{N}\right\}$ uma coleção de $N$ documentos que inclui $M$ termos $T=\left\{t_{1}, t_{2}, \ldots, t_{M}\right\}$. Cada documento $d_{i}$ é um vetor $v\left(d_{i}\right)=\left\{\right.$ freq $_{i 1}$, freq $_{i 2}, \ldots$, freq $\left._{i m\}}\right\}$, no qual o valor freq $_{i j}$ é alguma medida que determina a influência do termo $t_{j}$ no documento $\mathrm{d}_{\mathrm{i}}$, conforme representado na Tabela 1.
Tabela 1. Representação Vetorial.

\begin{tabular}{|c||c|c|c|c|}
\hline & $t_{1}$ & $t_{2}$ & $\cdots$ & $t_{M}$ \\
\hline \hline$d_{1}$ & freq $_{11}$ & freq $_{12}$ & $\cdots$ & freq $_{1 M}$ \\
\hline$d_{2}$ & freq $_{21}$ & freq $_{22}$ & $\cdots$ & freq $_{2 M}$ \\
\hline$\cdots$ & $\cdots$ & $\cdots$ & $\cdots$ & $\cdots$ \\
\hline$d_{N}$ & freq $_{N 1}$ & freq $_{N 2}$ & $\cdots$ & freq $_{N M}$ \\
\hline
\end{tabular}

Fonte: Alencar (2013).

A frequência freq $q_{i j}$ é calculada utilizando a medida terms frequency (tf). A medida considera $o$ valor de ocorrências de $t_{j}$ no documento $d_{i}$, sendo freq $q_{i j}=\operatorname{tf}\left(t_{j}, d_{i}\right)=$ freq $\left(t_{j}\right.$, $\left.d_{i}\right)$.

No presente trabalho, para o armazenamento dos termos e respectivas frequências, de cada documento, foi utilizada a estrutura de dados árvore rubro-negra, contando com sua eficiência na realização de buscas e ordenação de elementos com uma complexidade assintótica de $\mathrm{O}(\log (\mathrm{n}))$. Nesta fase do processamento, foi escolhida esta estrutura, pois é a etapa do préprocessamento que exige maior esforço computacional, visto que a cada termo retirado do texto deve-se verificar a lista de termos já extraídos e então caso já exista esse termo incrementa-se sua frequência, e caso seja um termo ainda não conhecido, adiciona-o na lista.

\subsection{DETERMINAÇÃO DAS FREQUÊNCIAS DOS TERMOS}

O objetivo principal da ferramenta TGEE é facilitar o entendimento de um conjunto de documentos por meio da técnica de 
visualização tag cloud, exibindo a frequência dos termos mais relevantes de cada documento desta coleção. A partir da visualização é possível analisar quais termos foram surgindo com o passar dos anos e então definir a evolução temporal sofrida pelos documentos.

É possível alternar facilmente pelas duas técnicas, WordCram e OpenCloud, implementadas na ferramenta. Utilizando a visualização WordCram é possível carregar uma imagem que dará forma a disposição dos termos no plano, sendo que a técnica verifica os limites desta imagem carregada, e projeta os termos no mesmo formato. Aconselha-se utilizar uma imagem preenchida de preto e com fundo branco, para facilitar a identificação dos limites da mesma. Essa técnica exibe os termos distinguindo-os pelo tamanho da fonte e diferença de cores, logo a ferramenta desenvolvida permite a definição de cores e limites de tamanhos máximos e mínimos que serão utilizados nas fontes dos termos. $\mathrm{Na}$ Figura 8 observa-se um modelo de imagem de entrada, no canto superior esquerdo, e, em uma escala maior, é exibida a saída de um processamento realizado utilizando $\mathrm{o}$ texto de resumo deste artigo. Também é possível utilizar a técnica OpenCloud, nesta os termos são dispostos em ordem crescente e suas frequências representadas pelo tamanho da fonte, também é possível definir uma cor para os termos e uma cor de fundo. Nesta visualização não é necessário carregar uma imagem de formato, o custo computacional é bem menor comparado ao da WordCram, pois não há necessidade de verificar limites de imagem, e nem tratar sobreposições dos termos.

Figura 8. Imagem de entrada e saída do processamento, utilizando a técnica WordCram, sobre o texto de resumo deste próprio artigo.

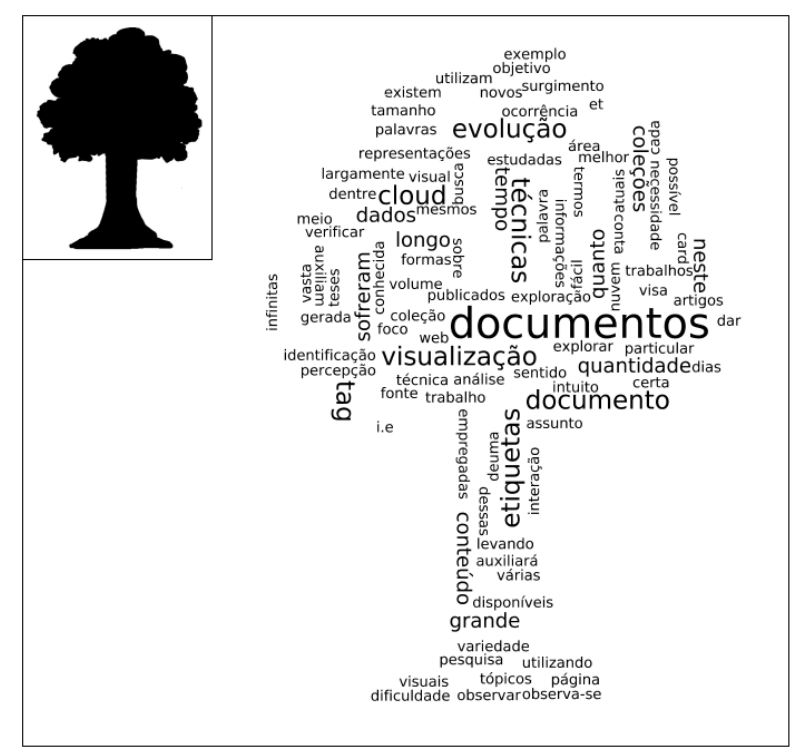

Fonte: (Própria).

A Figura 9 mostra o resultado do processamento utilizando novamente o texto do resumo deste artigo. Na ferramenta é possível definir o diretório de saída onde serão armazenados os resultados dos processamentos, visualizar o vetor de frequência de cada documento processado e as estatísticas do processamento realizado. 
Figura 9. Imagem de entrada e saída do processamento, utilizando a técnica OpenCloud, sobre o texto de resumo deste próprio artigo.

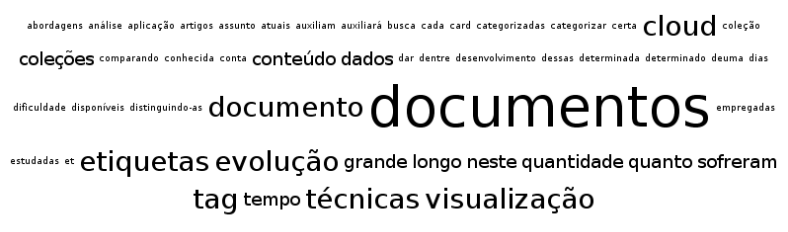

Fonte: (Própria).

\section{EXPERIMENTOS}

Para a validação da ferramenta TGEE descrita na seção anterior, foi analisada uma coleção de 96 trabalhos publicados pelos docentes do curso de Ciência da Computação da Faculdade de Ciência e Tecnologia (Unesp - Campus de Presidente Prudente, São Paulo, Brasil), nos últimos dez anos, de 2006 até 2016. As Figuras de 10 até 20 indicam o período analisado.

No ano de 2006 existe a publicação com o título: "Empowering ISO-surfaces with volume data" que trata especificamente de um algoritmo de visualização volumétrica denominado VoS - Volume on Surface. Observa-se na Figura 10 do respectivo ano, as palavras: "volume"; "surface"; e "rendering" em destaque. Mesmo sendo um único artigo, este utiliza uma alta frequência destes termos, desta forma os mesmos aparecem em destaque na visualização.

Percebe-se nas Figuras 11, 12 e 13, publicações dos anos 2007, 2008 e 2009, respectivamente, a alta frequência dos termos: "bone", "age", "skeletal" e "hand". Sendo que neste período existem vários trabalhos sobre métodos para estimativa da idade óssea, destacando-se a tese: "Novos métodos para estimativa da idade óssea baseado no processamento de imagens radiográficas da mão".

Existe na Figura 16, relacionada às publicações do ano de 2012, o destaque das palavras "algorithm" e "panoramic" sendo que neste ano existem duas publicações que tratam especificamente dos dois termos, sendo elas: "Construction of Panoramic Images from Aerial Images Obtained by Aircrafts" e "Um Novo Algoritmo para a Construção de Imagens Panorâmicas usando os algoritmos Sift e Ransac". Também, os termos "mappings", "multiple", e "fibers", relacionados a trabalhos de visualização.

Nos anos de 2013 e 2014, representados nas Figuras 17 e 18, respectivamente, observa-se em destaque 0 termo "recognition", e analisando os artigos publicados neste período são encontrados os seguintes títulos: "Deteç̧ão e Reconhecimento de Objetos em Imagens Utilizando Algoritmos de Extração De Pontos Chave"; "Reconhecimento de Objetos Coloridos e Mãos Usando Cores e Formas"; "Algoritmo para o Reconhecimento de Caracteres Manuscritos"; "Traffic Sign Detection and Recognition using then AdaBoost and SIFT algorithm"; e "Métodos 
para reconhecimento de notas musicais de partituras: resultados preliminares". Sendo que todos estes artigos tratam de técnicas de reconhecimento.

A Figura 21 apresenta o resultado do processamento unindo todos os trabalhos publicados no período de 2006 a 2016, sendo possível observar que os trabalhos publicados neste período utilizam os temas: "images"; "data"; "visualization"; e "recognition". Na Figura 22 o mesmo processamento é realizado, porém são adicionadas na lista de remoção as palavras comuns da coleção processada, e outras que não são relevantes e aparecem em quase todos os trabalhos, por exemplo: "work"; "approach", e "proposed". Essa remoção foi realizada em todos os processamentos realizados, visando destacar palavras mais relevantes. Com a visualização é possível ter uma visão geral dos assuntos abordados nesta coleção de documentos, indicando as principais linhas de pesquisas dos professores do curso de Ciência da Computação, sendo elas Visão Computacional e Visualização. Também pôde-se observar o surgimento de alguns tópicos em épocas específicas. Por meio da ferramenta esta análise é feita sem a necessidade de uma leitura completa de cada publicação, mesmo considerando a utilização do resumo e palavras chaves para facilitar a leitura, ainda assim verifica-se o quão trabalhoso seria realizar esta tarefa.

Figura 10. Tag Cloud gerada a partir de seis publicações dos docentes do curso de Ciência Computação da FCT no ano de 2006.

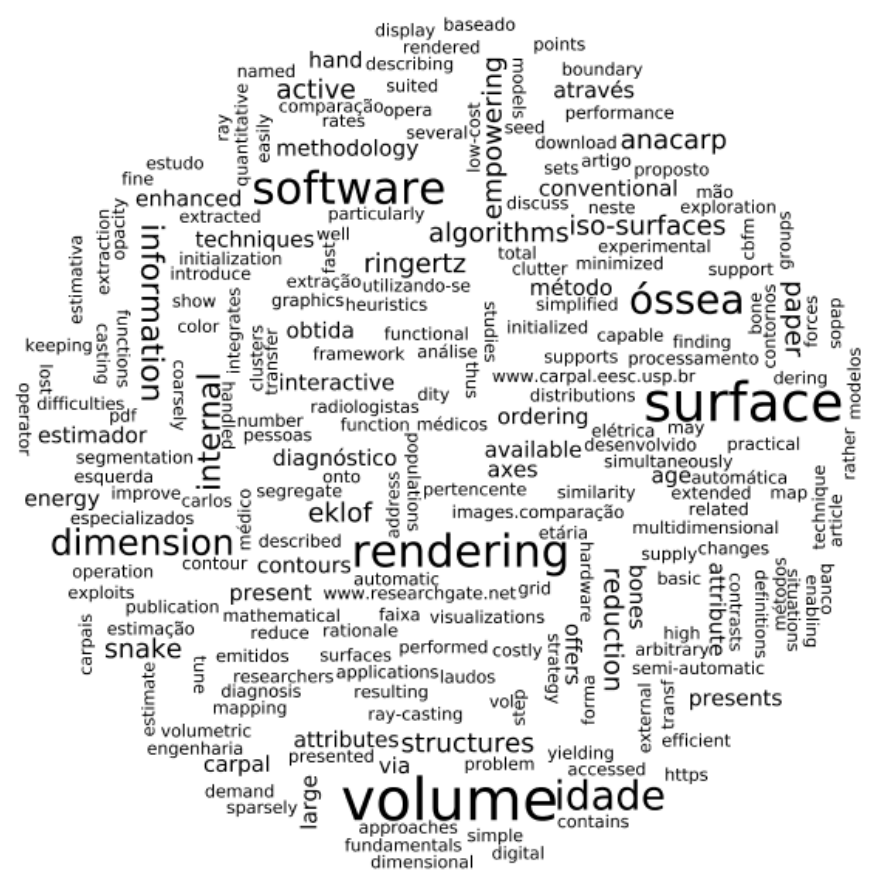

Fonte: (Própria). 
Figura 11. Tag Cloud gerada a partir de cinco publicações dos docentes do curso de Ciência Computação da FCT no ano de 2007.

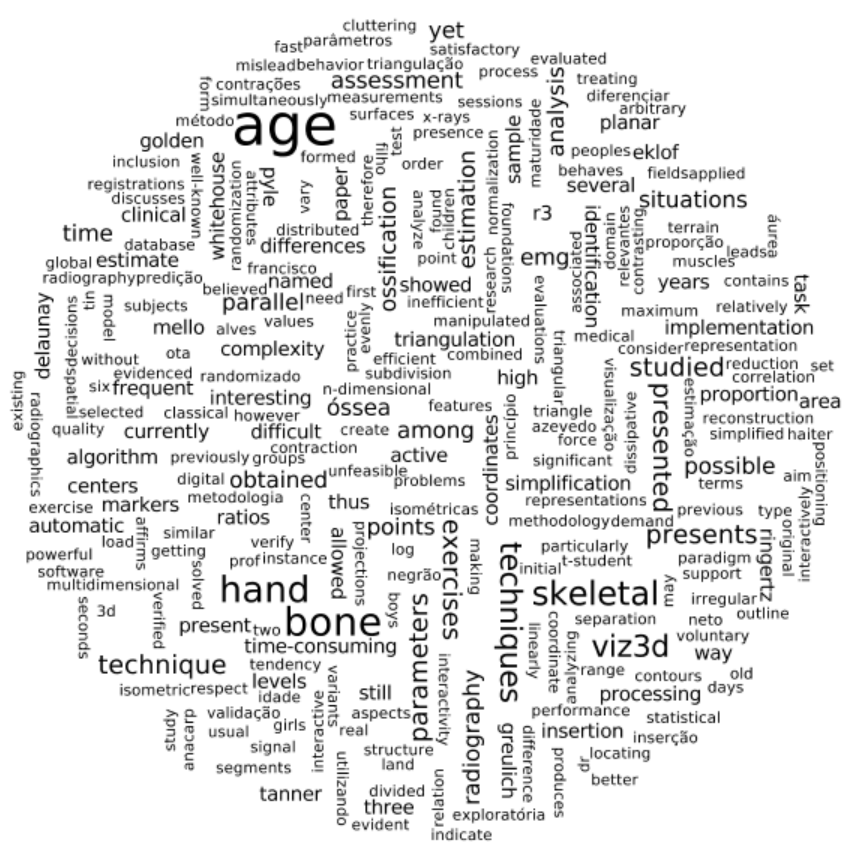

Fonte: (Própria).

Figura 12. Tag Cloud gerada a partir de três publicações dos docentes do curso de Ciência Computação da FCT no ano de 2008.

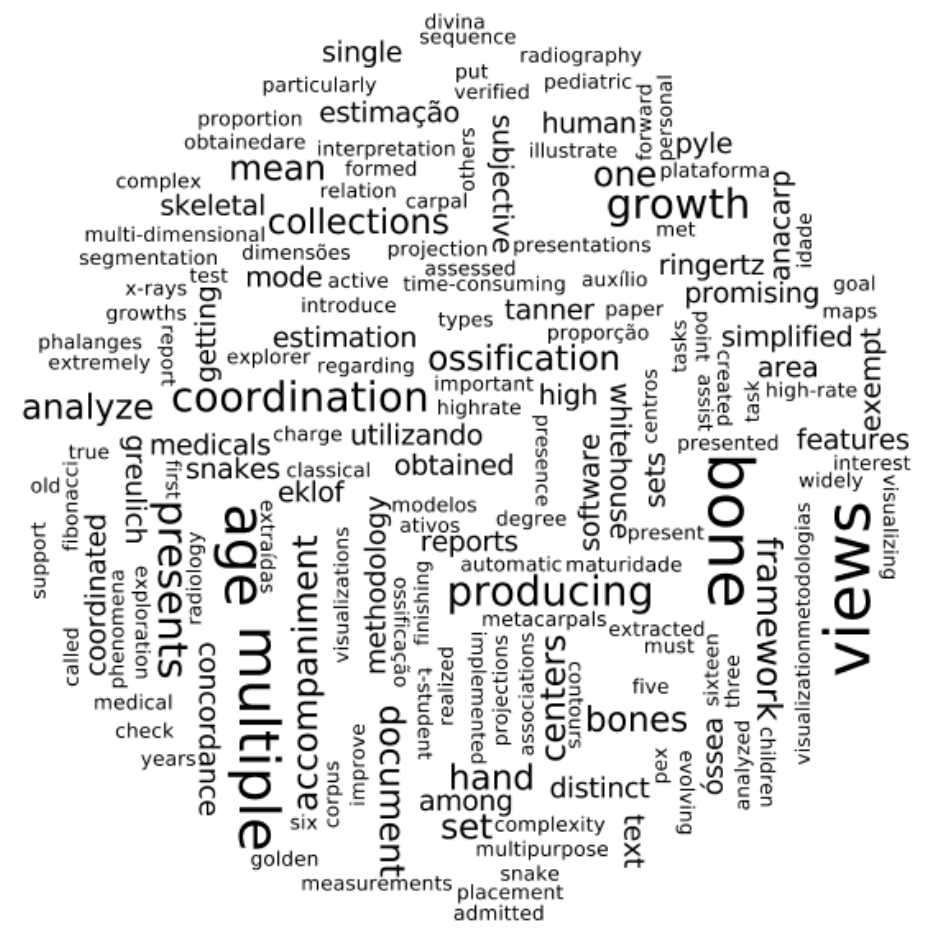

Fonte: (Própria). 
Figura 13. Tag Cloud gerada a partir de nove publicações dos docentes do curso de Ciência Computação da FCT no ano de 2009.

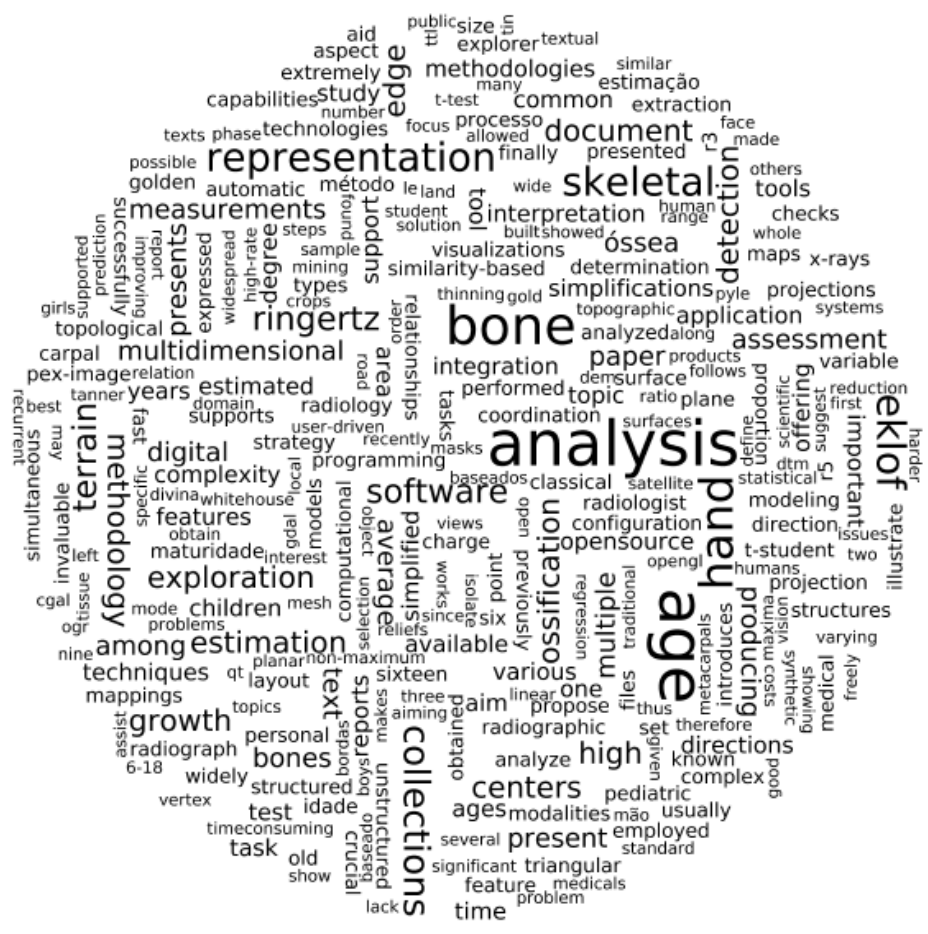

Fonte: (Própria).

Figura 14. Tag Cloud gerada a partir de 12 publicações dos docentes do curso de Ciência Computação da FCT no ano de 2010.

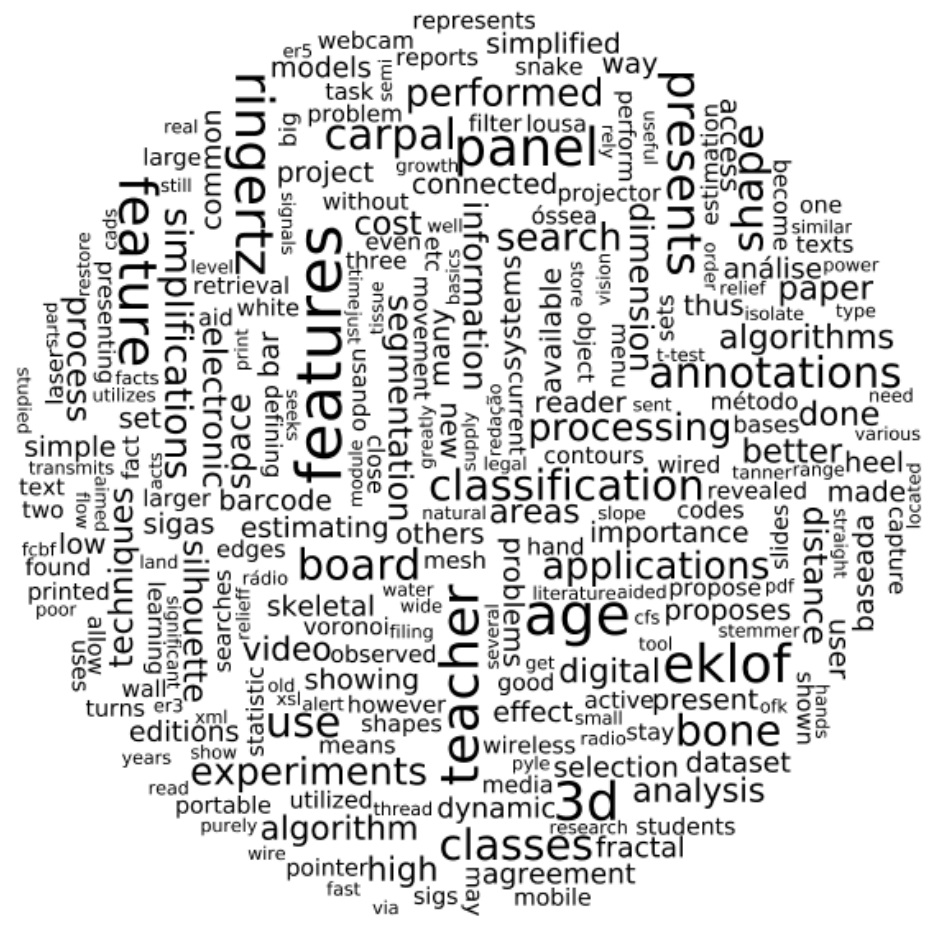

Fonte: (Própria). 
Figura 15. Tag Cloud gerada a partir de oito publicações dos docentes do curso de Ciência Computação da FCT no ano de 2011.

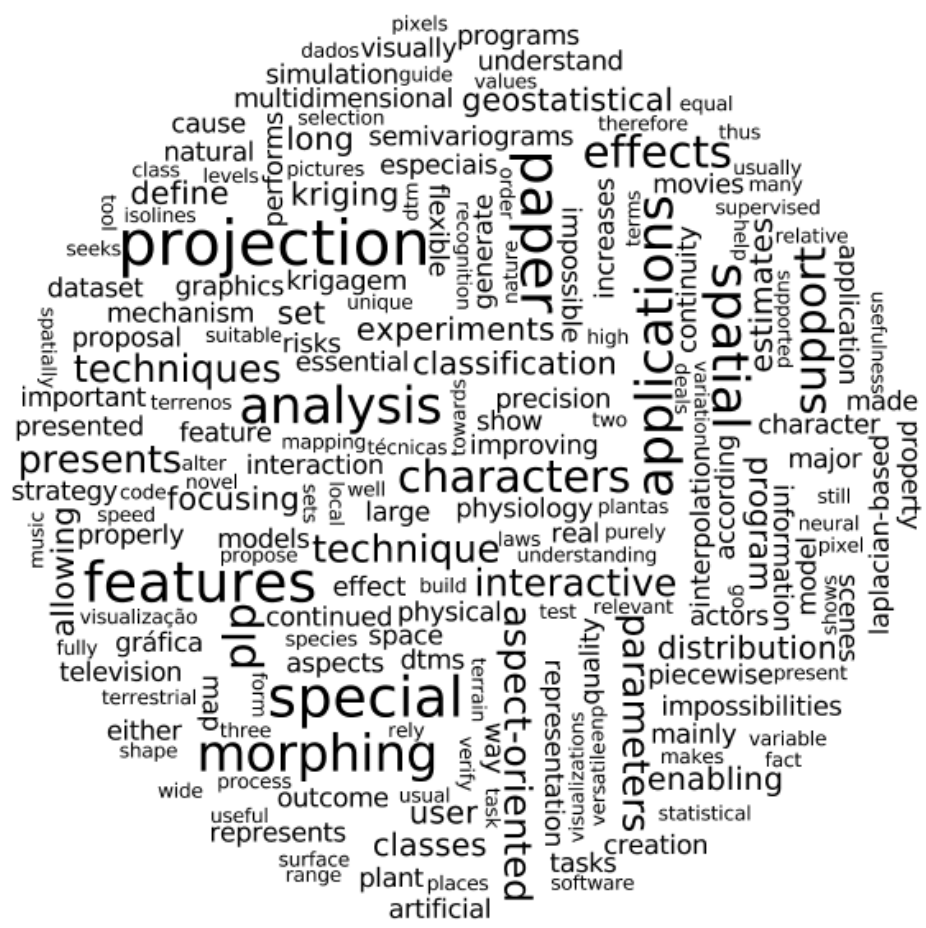

Fonte: (Própria).

Figura 16. Tag Cloud gerada a partir de nove publicações dos docentes do curso de Ciência Computação da FCT no ano de 2012.

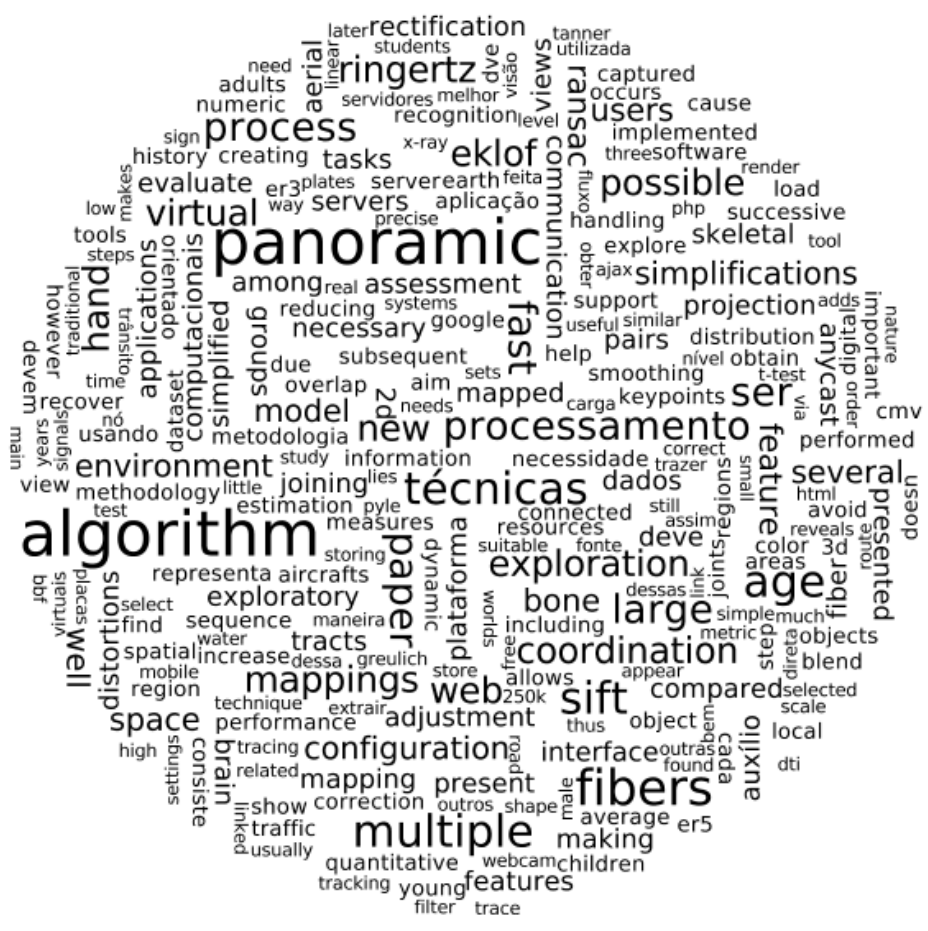

Fonte: (Própria). 
Figura 17. Tag Cloud gerada a partir de 13 publicações dos docentes do curso de Ciência Computação da FCT no ano de 2013.

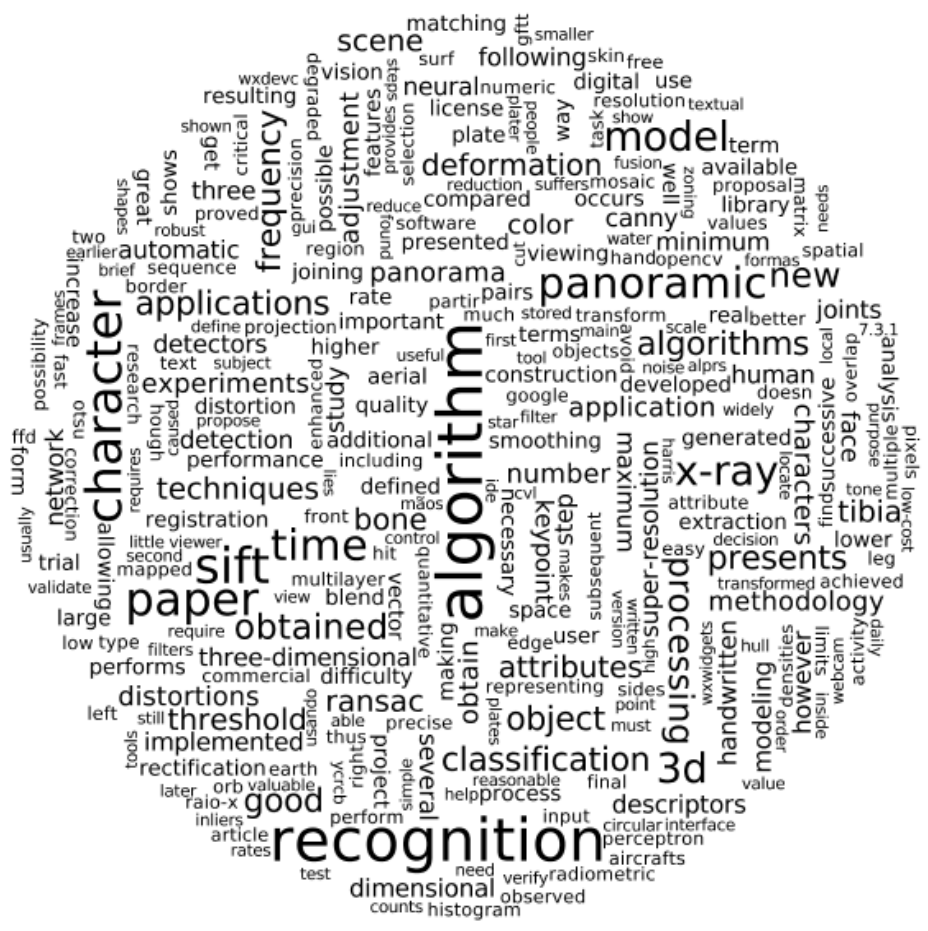

Fonte: (Própria).

Figura 18. Tag Cloud gerada a partir de 28 publicações dos docentes do curso de Ciência Computação da FCT no ano de 2014.

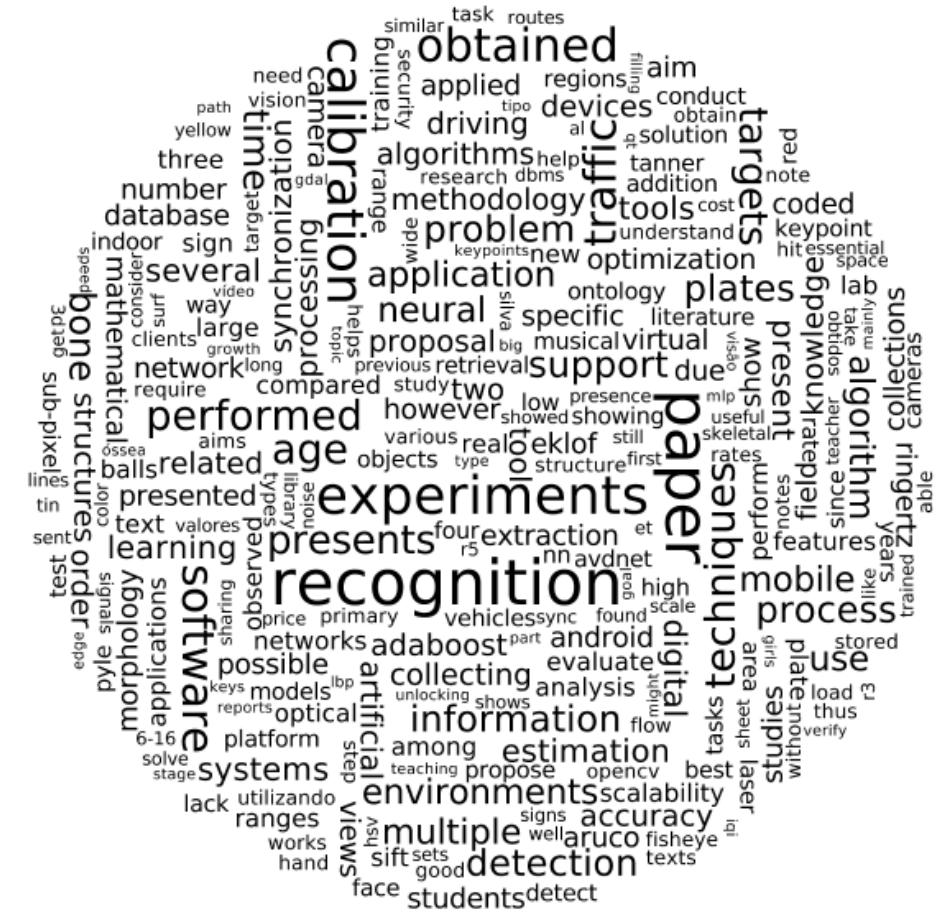

Fonte: (Própria.) 
Figura 19. Tag Cloud gerada a partir de uma publicação dos docentes do curso de Ciência Computação da FCT no ano de 2015.

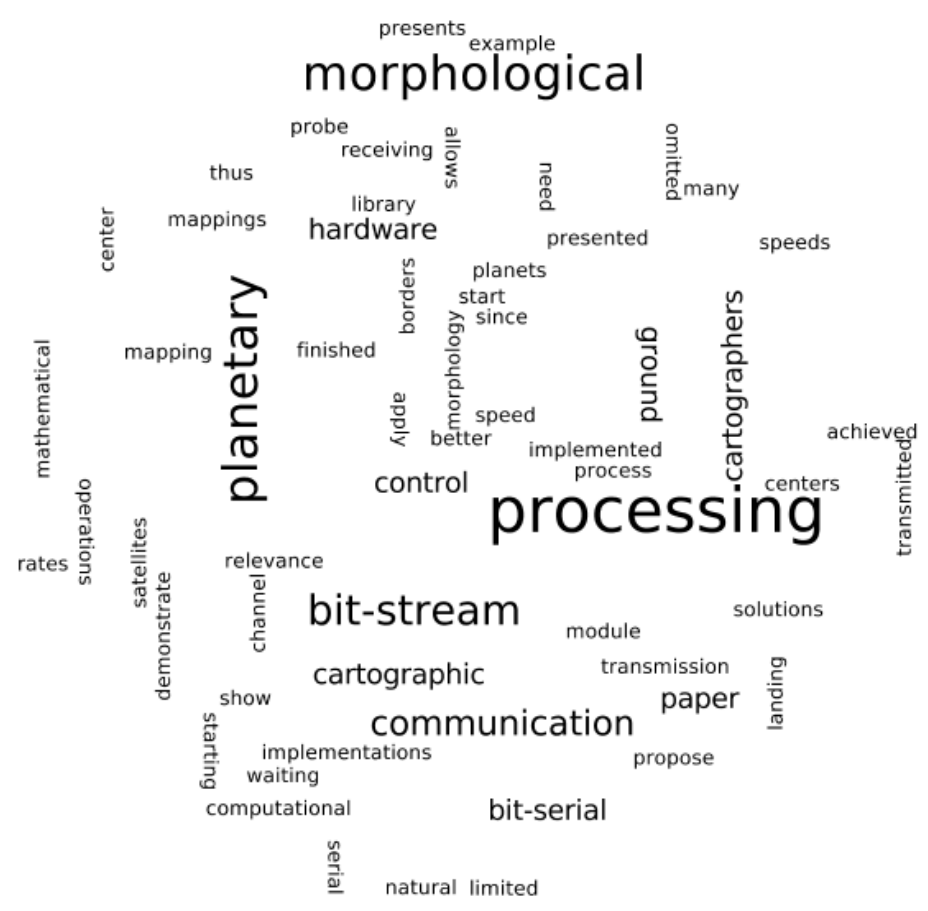

Fonte: (Própria).

Figura 20. Tag Cloud gerada a partir de duas publicações dos docentes do curso de Ciência Computação da FCT no ano de 2016.

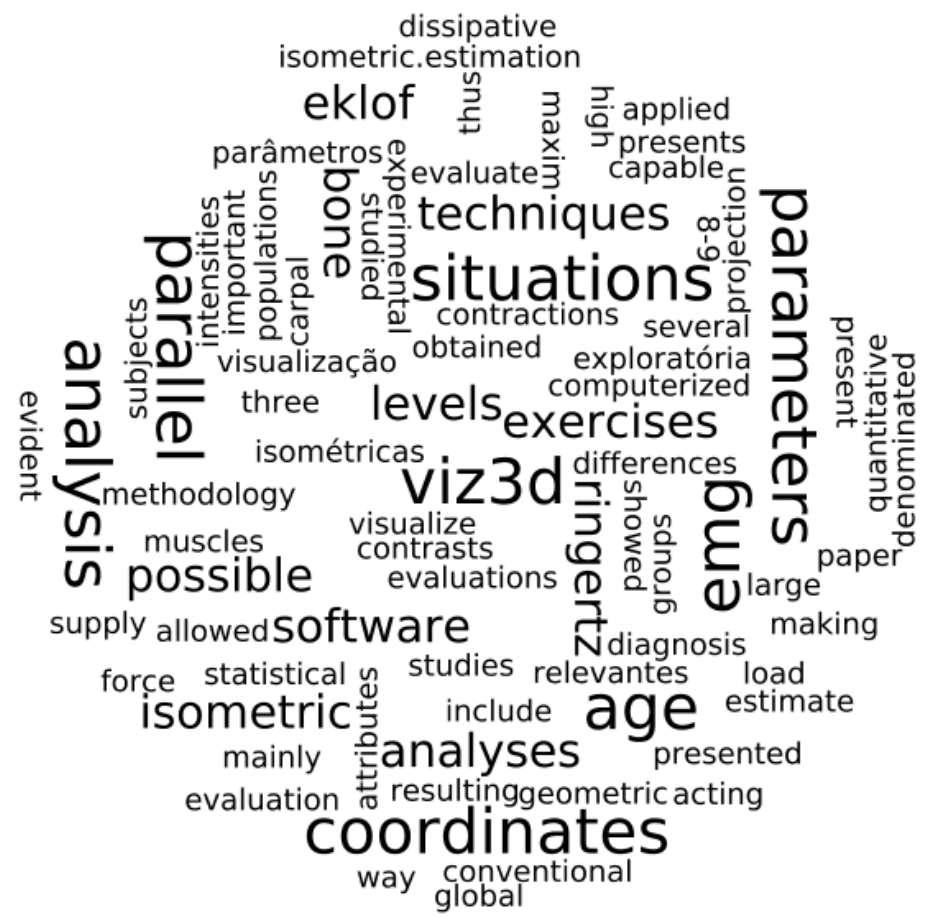

Fonte: (Própria). 
Figura 21. Tag Cloud gerada a partir de 96 publicações dos docentes do curso de Ciência Computação da FCT no período de 2006 a 2016.

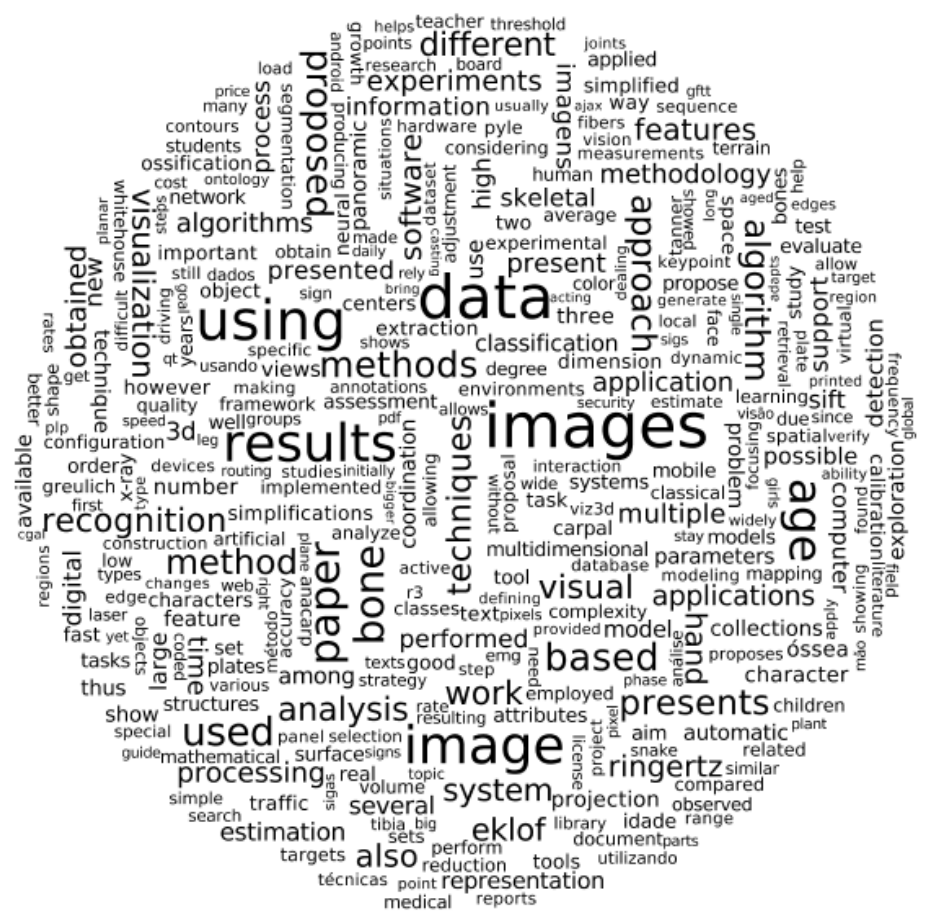

Fonte: (Própria).

Figura 22. Tag Cloud gerada a partir de 96 publicações dos docentes do curso de Ciência Computação da FCT no período de 2006 a 2016. Removendo palavras comuns da coleção e palavras irrelevantes.

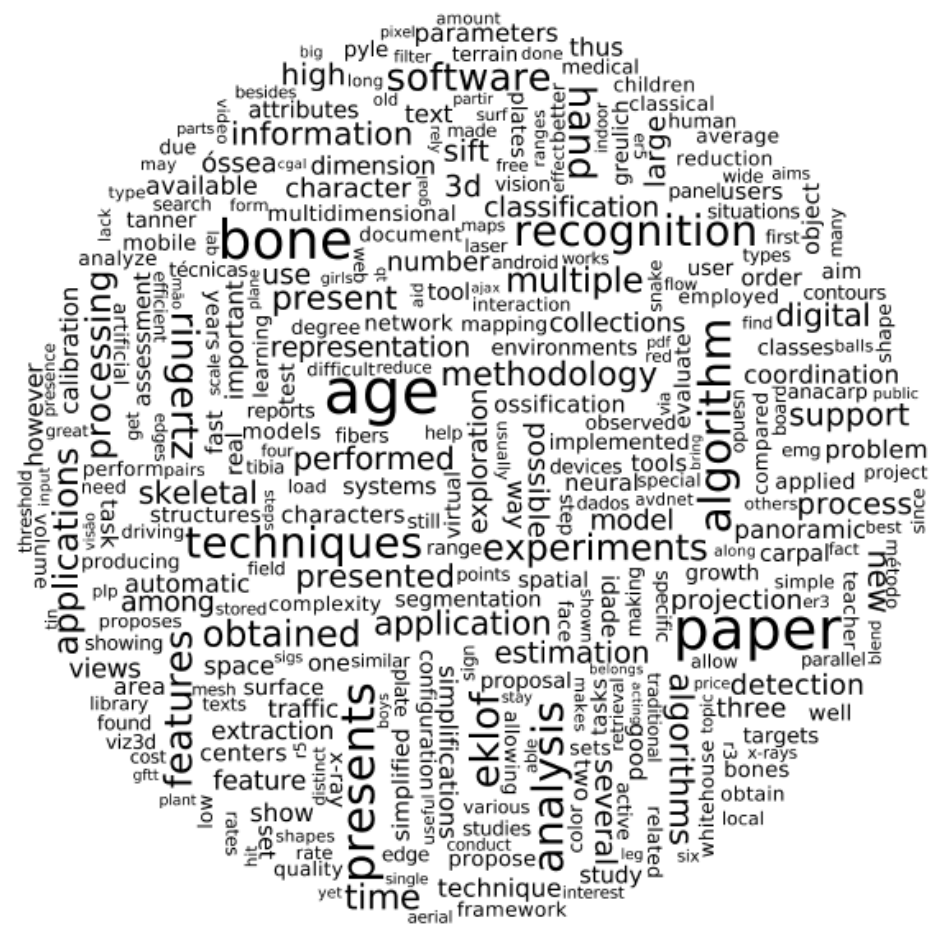

Fonte: (Própria). 


\section{CONCLUSÕES E TRABALHOS FUTUROS}

Neste trabalho foi estudada e aplicada a técnica de Visualização de Informação tag cloud na busca pela exploração de coleções de documentos e análise da evolução temporal sofrida por seus termos. Para a avaliação das técnicas investigadas foi implementada uma ferramenta, denominada TGEE, visando facilitar a exploração de coleções de documentos.

Para validação da ferramenta foi realizado o processamento numa coleção de artigos publicados pelos professores do curso de Ciência da Computação da Faculdade de Ciência e Tecnologia (Unesp - campus de Presidente Prudente). Pôde-se alcançar os resultados esperados, sendo possível verificar os principais tópicos pesquisados pelos docentes da computação do campus. 0 usuário pode escolher e alternar facilmente entre as duas técnicas implementadas, sendo elas WordCram e OpenCloud. Ainda persistem na visualização algumas palavras irrelevantes, porém a visualização ainda destaca as mais relevantes.

As duas técnicas utilizadas possuem suas particularidades, sendo a WordCram mais robusta, possuindo um visual elegante, possibilitando uma customização pelo usuário, por meio da escolha de imagem de formato, em contrapartida possui um maior custo computacional para a execução de seu processamento. Já a OpenCloud conta com uma visualização limpa, para o usuário que almeje uma visualização mais simples e um processamento menos custoso, exibi os termos em ordem alfabética, horizontalmente no plano, um após o outro.

Por meio dos experimentos realizados foi possível identificar que alguns pontos podem ser aperfeiçoados, por exemplo, algumas palavras irrelevantes que ainda persistem após o pré-processamento de remoção de palavras comuns, sendo que estas não são importantes para a análise do texto, também existem algumas palavras que estão presentes no documento tanto no plural quanto no singular, por exemplo, "image" e "images" na Figura 21, que poderiam ser unidas e representadas de forma única na visualização, aumentando suas frequências, levando em consideração que são equivalentes quanto ao seu significado.

Cabe ressaltar, que alguns termos aparecem com uma alta frequência em anos específicos, entretanto, quando se gera a tag cloud de toda a coleção, estes termos aparecem pequenos, ou nem aparecem, na visualização. Isso ocorre, pois, a frequência destes termos são relevantes em comparação aos demais termos do mesmo ano, porém, comparando-os com a coleção inteira possuem uma baixa frequência. Entretanto, pode-se ser implementada uma 
normalização, das quantidades por ano, destes termos, comparando a frequência máxima de cada ano e atribuindo um peso a cada termo, para que os mesmos tenham uma significância maior na tag cloud gerada para todos os anos.

Futuramente, a ferramenta poderá ser expandida para aceitar como entrada coleções de documentos em vários formatos que possuam larga utilização no meio acadêmico, por exemplo, ISI, Endnote Export Format ou BibTeX, facilitando a inserção dos dados. Também pode-se ser considerado a similaridade dos termos para unir termos que possuam o mesmo significado, e a utilização de uma matriz de covariância para detectar a frequência conjunta dos termos em todos os documentos.

\section{REFERÊNCIAS}

ALENCAR, A. B. Visualização da evolução temporal de coleções de artigos científicos. 2013. Tese (Doutorado) - Instituto de Ciências Matemáticas e de Computação (USP), São Carlos-SP, 2013.

CARD, S. K.; MACKINLAY, J. D.; SHNEIDERMANN, B. Readings in information visualizations: using vision to think. San Francisco, CA: Morgan Kaufmann, 1999. 712 p.

CHEN, C. Information visualization: beyond the horizon. Secaucus, NJ: Springer-Verlag, 2006.

KEIM, D. A. Information visualization and visual data mining. IEEE Transactions in Visualization and Computer Graphics, v. 8, n. 1 p. $1-8, \quad$ jan. 2002. https://doi.org/10.1109/2945.981847
LUHN, H. P. The automatic creation of literature abstracts. IBM Journal of Research and Development, Riverton, v. 2, n. 2, p. 159165, abr. 1958.

SALTON, G.; WONG, A.; YANG, C. S. A vector space model for automatic indexing. Communications of the ACM, New York, v. 18, n. 11, p. 613-620, nov. 1975. https://doi.org/10.1145/361219.361220

SEBASTIANI, F. Machine learning in automated text categorization. ACM Computing Surveys, v. 34 , n. 1 , p. 1-47, mar. 2002.

https://doi.org/10.1145/505282.505283

SPENCE, R. Information visualization: design for interaction. 2. ed. Harlow, England: Prentice Hall, 2007.

TAGCROWD visualization: State of the Union. 2011. Disponível em: < http://tagcrowd.com/blog/2011/03/05/state -of-the-union-2002-vs-2011/>.

WARRENA. Data cloud graph showing the closing percentage increase or decrease of the New York Stock Exchange. 2007.

ZIPF, G. K. Human behaviour and the principle of least effort: an introduction to human ecology. Cambridge, MA: AddisonWesley, 1949. $578 \mathrm{p}$. 